\title{
Estructura y composición florística de tres robledales en la región norte de la cordillera central de Colombia
}

\author{
Juan D. León ${ }^{1}$, Gladys Vélez ${ }^{1,2} \&$ Adriana P. Yepes ${ }^{3,4}$ \\ 1. Departamento de Ciencias Forestales, Universidad Nacional de Colombia, A.A. 1779, Medellín, Colombia; \\ jdleon@unalmed.edu.co \\ 2. gladys_velez@hotmail.com \\ 3. Ingeniera Forestal M.Sc. Universidad Nacional de Colombia, A.A. 1779, Medellín, Colombia. \\ 4. Centro de Investigación en Ecosistemas y Cambio Global - Carbono y Bosques. Calle 51A No. 72-23, interior 601. \\ Medellín, Colombia; adrianayepes@carbonoybosques.org
}

Recibido 20-XI-2008. Corregido 09-II-2009. Aceptado 13-III-2009.

\begin{abstract}
Structure and floristic composition of three oak forests in the northern region of the Central Cordillera in Colombia. Andean ecosystems harbor a high floristic diversity, which is being threatened by human disturbances such us deforestation and by the expansion of the agricultural frontier. One of these ecosystems are the Andean oak forests dominated by Quercus humboldtii, a threatened species in Colombia. We assessed the floristic composition and structure of three Andean oak forests located in three localities (San Andrés de Cuerquia, Belmira and Guarne) of Antioquia. The main goal was to determine whether these forests showed similarities in their structure and floristic composition. In each site, a permanent plot of $5000 \mathrm{~m}^{2}(0.5 \mathrm{ha})$ was established. All trees with $\mathrm{D} \geq 10 \mathrm{~cm}$ were sampled and identified to species. The Importance Value Index (IVI) was calculated as the sum of relative density (DeR), dominance (DoR) and frequency (FR) of a species. Trees with $5 \leq \mathrm{D}<10 \mathrm{~cm}$ and $2 \leq \mathrm{D}<5 \mathrm{~cm}$ were registered in sub-plots of 0.05 and 0.0144 ha, respectively. Finally, we used Jaccard's Index to quantify the floristic similarity among oak forest. When we considered all trees with $\mathrm{D} \geq 10 \mathrm{~cm}$ in the three forests, the number of species ranged from 18 to 54 , whilst the number of individuals ranged from 326 to 680 . The Guarne oak forest showed the highest species richness. In all sites, $Q$. humboldtii (Fagaceae) was the most important species in relation to the IVI, while Clusia sp. (Clusiaceae) and Myrsine coriaceae (Myrsinaceae) were the most important species in San Andrés de Cuerquia, Clethra fagifolia (Clethraceae) was important only in Belmira and Myrcia popayanensis (Myrtaceae) was important in Guarne. The families with the highest number of species were Fabaceae, Melastomataceae and Rubiaceae. Floristic similarity among places was low. The size distribution of trees had an inverse J-shape curve for all sites with changes in the abundance for size class. The three oak forests differed in their structure and floristic composition probably because of different disturbance degrees. Rev. Biol. Trop. 57 (4): 1165-1182. Epub 2009 December 01.
\end{abstract}

Key words: Andean forest, floristic composition, structure, oak forests, Quercus humboldtii, richness.

Los ecosistemas andinos son reconocidos como uno de los principales centros de diversidad y especiación en el mundo (Churchill et al. 1995, Brown \& Kapelle 2001). El norte de los Andes es uno de ellos, y se caracteriza por ser una de las regiones con la mayor diversidad de especies por unidad de área (Henderson et al. 1991). Sólo en el departamento de Antioquia (Colombia), que cubre alrededor de 63000 $\mathrm{km}^{2}$ existen aproximadamente 9000 especies de plantas. Esta diversidad se explica por la gran variabilidad geomorfológica y altitudinal que produce una alta complejidad de paisajes y climas, y en consecuencia, diversos tipos de bosque. No obstante, los bosques andinos son los ecosistemas forestales de Colombia más vulnerables a la conversión futura, ya que representan el $51 \%$ del área predicha a ser transformada (Etter et al. 2006, León 2008).

Dentro de estos, se encuentran los robledales, ecosistemas boscosos dominados por la especie Quercus humboldtii Bonpland., los 
cuales, a pesar de su gran potencial ecológico y florístico, se encuentran actualmente sometidos a fuertes presiones de origen antrópico, principalmente para la ampliación de la frontera agrícola (León \& Giraldo 1997). Específicamente, los robledales del departamento de Antioquia han estado sometidos a un proceso degradativo no solo a partir de la expansión de la frontera agrícola y pecuaria, la construcción de vías, la extracción comercial y la demanda de maderas como recurso genético, sino además, por la influencia de factores de índole regional como la minería, el proceso de curtición del cuero con extractos vegetales, y el ataque de plagas y enfermedades, entre otras causas (León \& Giraldo 1997). Las fuertes amenazas a las que se encuentra sometido el roble como especie característica de estos ecosistemas, y el desconocimiento elevado que se tiene de él y de sus relaciones específicas con otras especies, son algunas de las motivaciones para adelantar estudios que permitan dilucidar aspectos relacionados con su estructura y composición florística. El presente estudio ha tenido por objetivo caracterizar la estructura y la composición florística de tres robledales localizados en la Cordillera Central de Antioquia (Colombia), para lo cual han sido determinados diferentes parámetros de su estructura horizontal y diamétrica, así como su riqueza y composición florística. Los resultados se configuran en un referente de comparación para la valoración del estado de conservación de los escasos remanentes de robledal en el Departamento y del país, de los cuales y escasamente, ya existen reportes en la literatura especializada (Galindo et al. 2003, Pulido et al. 2006).

\section{MATERIALES Y MÉTODOS}

Área de estudio: El estudio se realizó en tres sitios diferentes de la Cordillera Central del Departamento de Antioquia, en jurisdicción de los municipios de Guarne (6 ${ }^{\circ} 16^{\prime} 42.073576$ " N, 75²9’35.670888” W), San Andrés de Cuerquia (6 $6^{\circ} 52^{\prime} 02.477617^{\prime \prime} \mathrm{N}, 75^{\circ} 35^{\prime} 59.910553^{\prime}$ ' W) y Belmira $\left(6^{\circ} 39^{\prime} 37.566876^{\prime \prime} \mathrm{N}\right.$, 75³9'12.029162" W).

Los bosques estudiados son ecosistemas complejos, dispuestos en zonas montañosas a gran elevación, de largas y fuertes pendientes, sobre suelos ácidos y desaturados, y sometidos a una alta precipitación y humedad (Cuadro 1).

CUADRO 1

Caracteristicas de los robledales de la Cordillera Central de Antioquia (Colombia)

TABLE 1

Characteristics from colombian Andean oak forests

\begin{tabular}{|c|c|c|c|c|c|}
\hline Sitio & Zona de vida & $\begin{array}{l}\text { Altitud } \\
\text { (m) }\end{array}$ & $\begin{array}{c}\text { Temperatura } \\
\left({ }^{\circ} \mathrm{C}\right)\end{array}$ & $\begin{array}{l}\text { Precipitación } \\
(\mathrm{mm})\end{array}$ & Suelos \\
\hline $\begin{array}{l}\text { San Andrés } \\
\text { de Cuerquia }\end{array}$ & bh-MB & 2820 & $12-18$ & $2550-3000$ & $\begin{array}{l}\text { Franco-arenosos. pH: 4.1. \%M.O: } 22.7 \text {. } \\
\text { Espesor de la capa de hojarasca: } 14 \mathrm{~cm} \text {. } \\
\mathrm{P}\left(\mathrm{mg} \mathrm{kg}^{-1}\right): 6 \text {; } \mathrm{Ca}\left(\mathrm{cmol}(+) \mathrm{kg}^{-1}\right): 0.2 \\
\mathrm{Mg}\left(\mathrm{cmol}(+) \mathrm{kg}^{-1}\right): 0.2 \\
\mathrm{~K}\left(\mathrm{cmol}(+) \mathrm{kg}^{-1}\right): 0.16\end{array}$ \\
\hline Belmira & bmh-MB & 2850 & $6-12$ & 2000 & 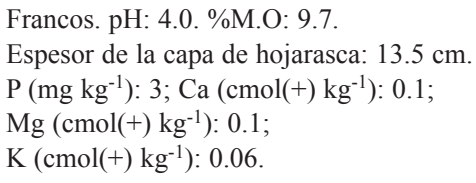 \\
\hline Guarne & bh-MB & 2400 & $13-17$ & 1612 & $\begin{array}{l}\text { Arcillosos. pH: 4.6. \%M.O: } 5.5 \text {. } \\
\text { Espesor de la capa de hojarasca: } 18 \mathrm{~cm} \text {. } \\
\mathrm{P}\left(\mathrm{mg} \mathrm{kg}^{-1}\right): 3 \text {; } \mathrm{Ca}\left(\mathrm{cmol}^{-}(+) \mathrm{kg}^{-1}\right): 0.1 ; \\
\mathrm{Mg}\left(\mathrm{cmol}^{-}(+) \mathrm{kg}^{-1}\right): 0.1 \\
\mathrm{~K}\left(\operatorname{cmol}(+) \mathrm{kg}^{-1}\right): 0.05\end{array}$ \\
\hline
\end{tabular}


Muestreo: En cada una de los sitios se estableció una parcela permanente con un núcleo básico de $50 \mathrm{~m} \mathrm{x} 100 \mathrm{~m}$ dentro del cual se trazaron al azar, al lado derecho del eje principal, cuatro fajas de $125 \mathrm{~m}^{2}(25 \mathrm{~m} \times 25 \mathrm{~m})$ con cinco unidades de registro de $5 \mathrm{~m} \times 5 \mathrm{~m}$ y tres subfajas al lado izquierdo de $48 \mathrm{~m}^{2}$ ( $24 \mathrm{~m}$ x $2 \mathrm{~m}$ ) con doce unidades de registro de $4 \mathrm{~m}^{2}$ ( $2 \mathrm{~m}$ x $2 \mathrm{~m}$ ). Dentro de cada unidad de registro, en las subfajas, se trazó un cuadro de $1 \mathrm{~m} \mathrm{x} 1$ $\mathrm{m}$ para evaluar la regeneración del roble. En el núcleo básico se marcaron, identificaron y midieron todos los árboles con diámetro normal (D) $\geq 10 \mathrm{~cm}$; en las fajas los individuos con $5 \leq \mathrm{D}<10 \mathrm{~cm}$, y en las subfajas, los árboles con $2 \leq \mathrm{D}<5 \mathrm{~cm}$. En los cuadros $\left(1 \mathrm{~m}^{2}\right)$ se realizó conteo de todos los individuos de roble menores de $30 \mathrm{~cm}$ de altura, entre $30 \mathrm{~cm}$ y $1 \mathrm{~m}$ de altura, y con altura mayor de $1 \mathrm{~m}$ y $\mathrm{D}<2$ $\mathrm{cm}$. Para todos los casos, se tomaron medidas como diámetro normal (D); diámetro, posición y calidad de copa; y altura total y altura de fuste. Adicionalmente, éstos se identificaron en el campo con la ayuda de un dendrólogo y para aquellos individuos sin identificación, se tomaron muestras botánicas para su identificación en el Herbario MEDEL de la Universidad Nacional de Colombia, Sede Medellín.

Procesamiento y análisis de la información: La estructura se evaluó a través de la riqueza, la composición florística, la estructura horizontal y la distribución diamétrica de la comunidad. La composición florística de especies con $\mathrm{D} \geq 10 \mathrm{~cm}$, se evaluó empleando la clasificación propuesta por Maleheiros \& Rotta (1982) que permite tipificar el comportamiento en abundancia de las especies presentes en los sitios. Especies representadas por 1-6 individuos corresponden a especies raras o escasas; aquellas con 7-25 árboles se consideran poco abundantes, y especies con más de 25 individuos se clasifican como abundantes. Adicionalmente, en cada bosque se calculó el índice de valor de importancia (IVI) como la sumatoria de la densidad relativa (DeR), la frecuencia relativa (FR) y la dominancia relativa (DoR) de las especies para evaluar la estructura horizontal del bosque (Matteucii \& Colma 1982); la dominancia se calculó empleando el área basal. La similitud florística entre los tres robledales, se determinó a partir del coeficiente de similitud de Jaccard, basado en la presencia/ ausencia de especies. Se evaluaron las distribuciones diamétricas de la especie $Q$. humboldtii construyendo histogramas de frecuencia del número de individuos por clase de tamaño y se ajustaron modelos que representaran confiablemente dicha distribución de tamaños (e.g. Weibull, Binomial negativa, Meyer I y Meyer II). Para el análisis de estos modelos, se tuvo en cuenta entre otros parámetros, el coeficiente de determinación $\left(\mathrm{R}^{2}\right)$, el análisis gráfico comparativo, el análisis de residuales y pruebas de bondad de ajuste.

\section{RESULTADOS}

Riqueza y composición florística: En Guarne se identificaron $45.9 \%$ de las morfoespecies presentes al nivel de especie (17), 45.9\% hasta género (17) y $8.1 \%$ hasta familia (tres). Para San Andrés de Cuerquia 70\% (siete) se clasificaron al nivel de especie y $30 \%$ (tres) hasta género. Para Belmira $47.1 \%$ se identificó hasta especie (ocho) y $52.9 \%$ hasta género. En total se encontraron 54 especies y 27 familias en Guarne, 18 especies y 13 familias en San Andrés de Cuerquia y, 37 especies y 14 familias en Belmira. En todos los casos el área efectiva muestreada fue de 0.5 ha.

El robledal de Guarne presentó mayor número de especies y familias, en comparación con los robledales de San Andrés de Cuerquia y Belmira. El número de árboles en las clases de tamaño superiores y el más bajo en las clases inferiores se presentó en Belmira (Cuadro 2). Empleando el sistema de clasificación propuesto por Maleheiros \& Rotta (1982) según la abundancia para los árboles con $\mathrm{D} \geq 10 \mathrm{~cm}$, en todas las regiones más del 50\% de las especies se clasificaron como escasas o raras, seguidas por las poco abundantes y las abundantes (Cuadro 3). En esta última categoría se encuentran especies como el roble ( $Q$. humboldtii), siendo exclusivo su carácter de abundante en San 
CUADRO 2

Número de especies, árboles y familias por hectárea en robledales de la Cordillera Central de Antioquia (Colombia)

TABLE 2

Species number, trees, and families per hectare in colombian Andean oak forests

\begin{tabular}{|c|c|c|c|c|c|c|c|}
\hline \multirow[b]{2}{*}{ Sitios } & \multicolumn{3}{|c|}{$\mathrm{S}$} & \multicolumn{3}{|c|}{$\mathrm{N}$} & \multirow[b]{2}{*}{$\mathrm{F}$} \\
\hline & $\begin{array}{c}0.5 \mathrm{ha} \\
\mathrm{D} \geq 10 \mathrm{~cm}\end{array}$ & $\begin{array}{c}0.05 \text { ha } \\
5 \leq \mathrm{D}<10 \mathrm{~cm}\end{array}$ & $\begin{array}{c}0.0144 \text { ha } \\
2 \leq \mathrm{D}<5 \mathrm{~cm}\end{array}$ & $\begin{array}{c}0.5 \mathrm{ha} \\
\mathrm{D} \geq 10 \mathrm{~cm}\end{array}$ & $\begin{array}{c}0.05 \text { ha } \\
5 \leq \mathrm{D}<10 \mathrm{~cm}\end{array}$ & $\begin{array}{c}0.0144 \text { ha } \\
2 \leq \mathrm{D}<5 \mathrm{~cm}\end{array}$ & \\
\hline $\begin{array}{l}\text { San Andrés } \\
\text { de Cuerquia }\end{array}$ & 10 & 6 & 10 & 326 & 180 & 1528 & 10 \\
\hline Belmira & 17 & 1 & 9 & 680 & 20 & 160 & 13 \\
\hline Guarne & 37 & 26 & 26 & 548 & 960 & 3333 & 28 \\
\hline
\end{tabular}

$\mathbf{S}=$ número de especies por hectárea. $\mathbf{N}=$ número de árboles por hectárea. $\mathbf{F}=$ número de familias por hectárea.

CUADRO 3

Distribución de las especies (S) según su abundancia (\%) en robledales de la Cordillera Central de Antioquia (Colombia)

TABLE 3

Species distribution (S) and abundance (\%) in colombian Andean oak forests

\begin{tabular}{|c|c|c|c|c|c|c|}
\hline \multicolumn{7}{|c|}{ Núcleo básico, árboles con $\mathrm{D} \geq 10 \mathrm{~cm}$} \\
\hline \multirow{2}{*}{ Categoría } & \multicolumn{2}{|c|}{ Guarne } & \multicolumn{2}{|c|}{ San Andrés de Cuerquia } & \multicolumn{2}{|c|}{ Belmira } \\
\hline & $S$ & $\%$ & $S$ & $\%$ & $S$ & $\%$ \\
\hline Raras & 22 & 60.1 & 9 & 90 & 9 & 50.0 \\
\hline Poco abundantes & 13 & 35.1 & 0 & 0 & 6 & 37.5 \\
\hline Abundantes & 2 & 5.4 & 1 & 10 & 2 & 12.5 \\
\hline
\end{tabular}

Andrés de Cuerquia, y compartiendo este comportamiento con otras especies en las parcelas restantes (e.g. Myrcia popayanensis Hieron. y Meriania nobilis Triana, en Guarne y Belmira, respectivamente).

En Belmira el número de especies por clase de abundancia fue relativamente homogéneo, dado el bajo grado de intervención que presumiblemente tiene el bosque. Para los tres sitios, el roble fue la especie más abundante, representando 49.3\% en Guarne, 90.8\% en San Andrés de Cuerquia y $77.6 \%$ en Belmira. $M$. popayanensis (arrayán) también se clasificó dentro de la categoría de abundante en Guarne, y Meriania sp. en Belmira. En términos de la riqueza por familias, en Guarne se encontraron 22 familias, la mayoría representadas por una o tres especies (Anexo 1). La familia con mayor número de especies fue Fabaceae (139), seguida por Myrtaceae (19), Lauraceae (14) y Clusiaceae (13). En Belmira se encontraron 12 familias de las cuales, más del $75 \%$ estuvieron representadas por una sola especie. Melastomataceae (cuatro), Rubiaceae (dos) y Theaceae (dos) fueron las familias con mayor número de especies. Para San Andrés de Cuerquia, se registraron nueve familias de las cuales solo una presentó dos especies (Clusiaceae), las restantes estuvieron representadas por una.

Con respecto a las especies arbóreas de 2 $\leq \mathrm{D}<10 \mathrm{~cm}$, en Guarne se presentó el número más alto de individuos por hectárea. Sin embargo, $88 \%$ de las especies no estuvieron representadas en las clases superiores (Anexo 
1); sólo Dendropanax sp., Geissanthus sp. y M. popayanensis fueron encontradas en todas las clases de tamaño muestreadas. Las especies que presentaron mayor número de individuos (cinco) fueron Alfaroa colombiana Lozano, Hern. Cam. \& Espinal., Palicourea sp1., Palicourea sp2., M. popayanensis y Symplocos rigidissima Brand.; las demás, presentaron solo un individuo. Adicionalmente se encontraron otras familias como Boraginaceae, Actinidiaceae, Caprifoliaceae, Staphylaceae, Piperaceae y Melastomataceae. Únicamente en San Andrés de Cuerquia el roble estuvo representado en todas las categorías de tamaño; no obstante, el número de individuos en las clases de tamaño entre $2 \leq$ $\mathrm{D}<5 \mathrm{~cm}$ fue bajo. Las especies más abundantes en este sitio fueron Cavendishia pubescens (Kunth) Hemsl (ocho), Graffenrieda sp. (tres), Schefflera sp. (dos) y Miconia sp1. (dos).

Estructura horizontal: En los tres sitios, el roble fue la especie dominante presentando valores de importancia de $44.6 \%$ en Guarne, $61.1 \%$ en San Andrés de Cuerquia y $77.2 \%$ en Belmira (Anexo 2). Esto confirma la dominancia florística de esta especie sobre las demás, en especial en San Andrés de Cuerquia. Las especies comunes en todas las regiones no presentaron la misma importancia ecológica. Por ejemplo, Clethra fagifolia Kunth, que fue la segunda especie más abundante en Belmira, tuvo bajos valores en Guarne y San Andrés de Cuerquia, siendo sus porcentajes con respecto al total en cada sitio de: $5.8 \%, 2.3 \%$ y $1.8 \%$ respectivamente. Por su parte, Weinmannia pubescens Kunth, mostró mayor importancia en Belmira, luego en Guarne y por último en San Andrés de Cuerquia. En Guarne la segunda especie más importante desde el punto de vista ecológico fue M. popayanensis $(\mathrm{IVI}=16.4)$ seguida por Ilex laurina Kunth (IVI $=11.3)$ y, en San Andrés de Cuerquia, Clusia sp. (IVI = 11.9) y Myrsine coriacea (Sw.) R. Br. (IVI = 11.6).

Similitud entre sitios: Para los árboles con $\mathrm{D} \geq 10 \mathrm{~cm}$, la mayor similitud se encontró entre las parcelas de San Andrés de Cuerquia y Belmira, las cuales compartieron alrededor de $21 \%$ de las especies. Por su parte, los robledales de Guarne compartieron igual porcentaje de especies con los robledales de San Andrés de Cuerquia y Belmira (14\%). Para las especies arbóreas con diámetro entre 5 y $10 \mathrm{~cm}$, la disimilitud fue de $100 \%$. Las parcelas no compartieron especies en esta clase de tamaño. La mayor similitud entre los sitios para los árboles con $2 \leq \mathrm{D}<5 \mathrm{~cm}$, se presentó entre Guarne y San Andrés de Cuerquia, ambos sitios compartieron 2 especies que corresponden a $5.8 \%$ (Cuadro 4).

CUADRO 4

Índices de similitud de Jaccard para los robledales de la Cordillera Central de Antioquia (Colombia)

TABLE 4

Jaccard Index for colombian Andean oak forests

\begin{tabular}{|c|c|c|c|}
\hline & Guarne & San Andrés de Cuerquia & Belmira \\
\hline \multicolumn{4}{|c|}{$\mathrm{D} \geq 10 \mathrm{~cm}$} \\
\hline Guarne & 1.00 & 0.14 & 0.14 \\
\hline San Andrés de Cuerquia & & 1.00 & 0.21 \\
\hline Belmira & & & 1.00 \\
\hline \multicolumn{4}{|c|}{$5 \leq \mathrm{D}<10 \mathrm{~cm}$} \\
\hline Guarne & 1.00 & 0.00 & 0.00 \\
\hline San Andrés de Cuerquia & & 1.00 & 0.00 \\
\hline Belmira & & & 1.00 \\
\hline \multicolumn{4}{|c|}{$2 \leq \mathrm{D}<5 \mathrm{~cm}$} \\
\hline Guarne & 1.00 & 0.06 & 0.00 \\
\hline San Andrés de Cuerquia & & 1.00 & 0.00 \\
\hline Belmira & & & 1.00 \\
\hline
\end{tabular}


Distribuciones diamétricas: Los modelos que mejor representaron la distribución por clases de tamaño diamétrico del roble, fueron la Weibull y la Binomial negativa $\left(\mathrm{R}^{2}>0.95\right.$; Cuadro 5). En San Andrés de Cuerquia, el número total de árboles de roble fue de 296. Las clases de tamaño inferiores estuvieron escasamente representadas, mientras que la clase de tamaño con diámetro igual a $42.5 \mathrm{~cm}$, fue la mejor representada con 16 individuos (Fig. 1A). Para Belmira, la distribución diamétrica mostró una tendencia inicial creciente para las dos primeras marcas de clase, y luego comenzaron a decrecer los valores de frecuencia (Fig. 1B). El número total de árboles de roble fue de 548 y la clase mejor representada correspondió a la de 42.5 $\mathrm{cm}$ de diámetro con 14 árboles. Finalmente para Guarne, se encontraron en total 270 árboles de roble, siendo éste el valor más bajo de todas las parcelas. La distribución mostró alta concentración de valores en los tamaños intermedios (diámetro $22.6 \mathrm{~cm}$ ) con un descenso en las clases siguientes (Fig. 1C).

Para los tres sitios la distribución por clases de tamaño se ajustó a la forma de J invertida, con árboles que alcanzaron tamaños considerables (e.g. $>50 \mathrm{~cm}$ ). Sin embargo, la presencia de árboles con $1 \leq \mathrm{D}<10 \mathrm{~cm}$ fue escasa o nula, lo que sugiere una incipiente regeneración de esta especie.

\section{DISCUSIÓN}

Riqueza y composición florística: Por su composición florística, los bosques estudiados corresponden a bosques típicos de la formación andina, que se caracterizan por presentar especies con dominancia notoria (Cuatrecasas 1958). En términos de riqueza de especies, los resultados obtenidos sugieren que ésta fue baja en San Andrés de Cuerquia y Belmira, si se compara con los registros citados para otros bosques de la región tropical, donde el número de especies reportado varía entre 43 y 91 (Cuadro 6). Caso contrario ocurre para el robledal de Guarne, cuya riqueza sí está dentro del rango reportado para la región tropical (Vélez \& Fresneda 1992, Gentry 1995, Marín-Corba $\&$ Betancur 1997). De esta manera, las diferencias encontradas entre los robledales del norte y centro de Antioquia en términos de riqueza de especies, podrían explicarse por características particulares del sitio como la historia de uso de los mismos, el tipo e intensidad de las perturbaciones pasadas, la topografía del lugar y la fauna existente. Estos factores podrían ser claves en el desarrollo de la dinámica forestal (e.g. tasa de formación de claros, regeneración natural, dispersión, entre otros) (Aide et al. 1995, Finegan 1996, Guariguata \& Ostertag 2001, Kennard 2001) y verse reflejados en

CUADRO 5

Modelos ajustados y los estadísticos asociados para las distribuciones diamétricas de los robledales de la Cordillera Central de Antioquia (Colombia)

TABLE 5

Adjusted models and associated statistics for diametric distribution from colombian Andean oak forests

\begin{tabular}{lcccc} 
Sitio & Modelo & $\mathrm{R}^{2}$ & \multicolumn{2}{c}{ K-S } \\
San Andrés de Cuerquia & Weibull & 0.9880 & 0.2000 & 0.9900 \\
Belmira & Binomial negativa & 0.9600 & 0.2000 & 0.98826 \\
Guarne & Weibull & 0.9680 & 0.2200 & 0.9790
\end{tabular}

K-S: prueba de bondad de ajuste Kolmogorov-Smirnof 

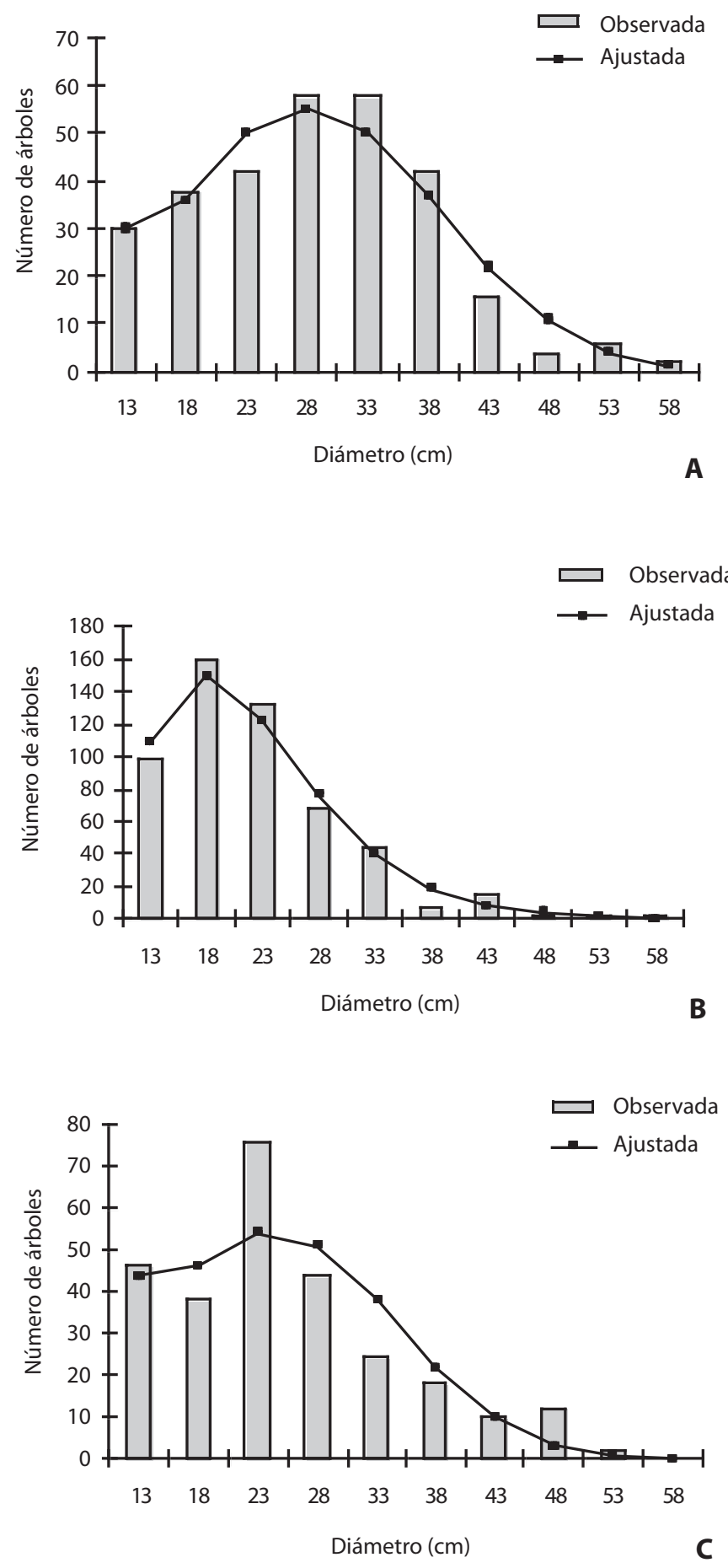

Fig. 1. Distribuciones diamétricas para robledales de la Cordillera Central de Antioquia (Colombia). (A) San Andrés de Cuerquia, (B) Belmira y (C) Guarne.

Fig. 1. Diametric distributions for colombian Andean oak forests. (A) San Andrés de Cuerquia, (B) Belmira and (C) Guarne. 
CUADRO 6

Comparación de la riqueza florística en diferentes bosques andinos. Modificado de Galindo et al. 2001

TABLE 6

Comparative floristic richness between different Andean forests. Modified from Galindo et al. 2001

$\begin{array}{lccccc}\text { Sitio } & \begin{array}{c}\text { Altitud } \\ (\mathrm{m})\end{array} & \begin{array}{c}\text { No. } \\ \text { Familias }\end{array} & \begin{array}{c}\text { No. } \\ \text { Especies }\end{array} & \begin{array}{c}\text { No. } \\ \text { Individuos }\end{array} & \text { Fuente } \\ \text { P.N.N. Iguaqué, Boyacá-Colombia } & 2800 & 27 & 53 & 384 & \text { Marín-Corba y Betancur (1997) } \\ \text { Sacramento, Bolivia } & 2450 & 33 & 91 & 572 & \text { Gentry (1995) } \\ \text { Cerro Espejo, Guajira } & 2500 & 46 & 78 & 406 & \text { Gentry (1995) } \\ \text { Cerro Kennedy, Magdalena } & 2550 & 35 & 57 & 326 & \text { Gentry (1995) } \\ \text { La Sierra, Santander } & 2500 & 40 & 85 & 411 & \text { Marín-Corba y Betancur (1997) } \\ \text { El Venado, Boyacá } & 3100 & 23 & 43 & 605 & \text { Marín-Corba y Betancur (1997) } \\ \text { San Andrés de Cuerquia } & 2775 & 13 & 18 & 194 & \text { Este estudio } \\ \text { Belmira } & 2450 & 14 & 37 & 354 & \text { Este estudio } \\ \text { Guarne } & 2455 & 27 & 54 & 349 & \text { Este estudio }\end{array}$

características del bosque como la riqueza y la composición florística. Por ejemplo, algunos estudios han planteado que el número de especies por unidad de área, aumenta en proporción inversa a la calidad del suelo (IDEADE 1996). Analizando los resultados, puede observarse que San Andrés de Cuerquia es el sitio con mayor contenido de materia orgánica en el suelo y nutrientes (Cuadro 1), pero al mismo tiempo fue el que presentó el número de especies más bajo, en comparación con Belmira y Guarne. Por su parte, la alta riqueza de este último, ocurrió en suelos con los más bajos contenidos de materia orgánica y bajas concentraciones de elementos que son importantes para la nutrición vegetal. De esta manera, la hipótesis según la cual la riqueza de especies varía inversamente con la calidad de los suelos es factible, y debería ser evaluada puntualmente en futuros estudios, para obtener resultados contundentes con relevancia estadística.

La historia de los sitios también parece ser un factor determinante en la composición florística y la riqueza de especies, por cuanto, las perturbaciones localizadas según su frecuencia, tamaño y duración pueden inducir o afectar la regeneración natural de los sitios y la ocurrencia de especies (Denslow 1980, Hartshorn 1980, Martínez-Ramos 1985, Coomes \& Allen
2007). En el caso de Guarne, por ejemplo, se tienen registros de colonización a partir de principios del siglo XVII, cuando las personas comenzaron a agruparse en los alrededores de Guarne para dedicarse a la minería de aluvión (Vélez \& Botero 2000). Aunque actualmente todavía pueden apreciarse las grandes cárcavas producidas por el laboreo de las minas para el beneficio, remoción y lavado del material aurífero, estas prácticas pudieron de alguna manera estimular la regeneración de algunas especies de plantas, lo que favoreció la mayor riqueza florística del lugar comparada con la de San Andrés de Cuerquia y Belmira, donde al parecer, los bosques son más conservados. De hecho, el cambio en la composición de la avifauna en la zona durante las últimas décadas (Castaño \& Patiño 2000) puede ser el resultado de la dinámica de fragmentación que se dio en la zona, y que consecuentemente pudo incidir de manera negativa o positiva en la llegada y permanencia de especies vegetales que dependen de cierto grupo trófico de aves para su dispersión. Estudios al respecto son necesarios para validar estas suposiciones, y encontrar relaciones ecológicas de dependencia entre las especies de aves y de flora existentes. En síntesis, la composición florística y la riqueza de especies evaluadas, sugieren diferencias 
significativas entre los robledales estudiados que parecen depender de las características particulares de los sitios, aún cuando de manera general, dichos ecosistemas exhiban condiciones climáticas similares.

El hecho de que el roble haya sido la especie más abundante en los tres sitios estudiados, coincide con algunos de los estudios realizados en bosques andinos (Vélez \& Fresneda 1992, Galindo et al. 2003). Sin embargo, las demás especies abundantes después de $Q$. humboldtii, varían en la mayoría de ellos. Por ejemplo, Chusquea sp. y Cavendishia sp. fueron citadas como las especies más abundantes después del roble, en una sucesión secundaria de 16.5 años en Duitama-Boyacá (Becerra 1989), mientras que para robledales situados al norte de la Cordillera Oriental, se reportaron especies como Clusia sp. (gaqui), Weinmannia tomentosa (encenillo), Hedyosmum sp. (granizo), Cyathea sp. (helecho arbóreo) y algunas pertenecientes a la familia Rubiaceae (IDEADE 1996). En el Santuario de Flora y Fauna GuanentáRío Fonce (Cordillera Central de Colombia), especies como Centronia dichromantha L. Uribe., Clethra lanata M. Martens \& Galeotti., Chusquea aff. lehmanni y Graffenrieda uribei Wurdack., fueron las especies más abundantes después del roble, para los cuatro sitios muestreados (Galindo et al. 2003). En el presente estudio, especies como Meriania sp., M. popayanensis y A. colombiana Lozano, Hern. Cam. \& Espinal., fueron las más abundantes después de roble. Estos resultados sugieren que la variación en las abundancias de las especies entre sitios, puede estar relacionada con el grado de intervención local, los patrones de dispersión de especies y otras características particulares de los sitios que pueden condicionar el establecimiento y permanencia de las especies (Galindo et al. 2003). Incluso para el caso del $Q$. humboldtii, se sabe que su abundancia es mayor en las laderas secas de las cordilleras, especialmente en la ladera occidental de la Cordillera Oriental, donde las características biofísicas favorecen el crecimiento y regeneración de esta especie (Marín-Corba \& Betancur 1997, Cavelier et al. 2001).
Beta diversidad: El porcentaje de especies con $\mathrm{D} \geq 10 \mathrm{~cm}$ comunes entre las distintas regiones fue bajo; esto confirma la diferencia que existe entre estos ecosistemas en términos de la variedad de especies y de su composición florística; las diferencias en similitud de especies entre parcelas, reflejan alta diversidad beta ya que la composición florística de cada una de ellas, puede depender de varios factores como la amplitud de destrucción de la vegetación, la flora de los alrededores, los agentes dispersores y los usos previos de la tierra (Fontaine et al. 1980), los valores del IVI obtenidos y el índice Jaccard así lo demuestran. Desde esta perspectiva, las diferencias florísticas entre los tres sitios muestreados, puede ser el resultado del alto grado de endemismos que presentan los bosques andinos (Gentry 1995), por ello, cada ecosistema tiene y requiere estrategias de protección y recuperación propias que se ven reflejadas en la composición florística que exhiben. Estudios más detallados a largo plazo, que involucren la dinámica y desarrollo de los mismos, deben ser desarrollados para entender la complejidad de su funcionamiento y los patrones que éstos exhiben.

Estructura horizontal: Según los resultados, se concluye que el roble es la especie dominante en los tres ecosistemas evaluados; resultados análogos fueron reportados para robledales del departamento de Boyacá, donde la abundancia relativa para $Q$. humboldtii fue de $67.3 \%$, valor equivalente al encontrado para las abundancias en las parcelas de este estudio. En este contexto, el término de robledales es propio para los sitios muestreados y comprueba al igual que en otros estudios, la existencia de una o varias consociaciones en las cuales el roble es la especie dominante (Kapelle 2001). Este hecho pone de manifiesto que ante las grandes amenazas a las que se enfrenta esta especie, su importancia ecológica, el desconocimiento de su fenología y las relaciones interespecíficas que pueda tener con la fauna local, es necesario continuar con la realización de estudios que permitan diseñar estrategias de manejo para su conservación futura. 
Distribuciones diamétricas: Los modelos ajustados para las tres regiones, muestran que las distribuciones por clases de tamaño del $Q$. humboldtii tienden a la forma de $\mathrm{J}$ invertida, en la cual, las primeras clases de tamaño se encuentran representadas por un alto número de individuos que posteriormente decrece conforme aumenta el tamaño de los árboles; esta forma de distribución representa la mejor garantía para que una población se mantenga equilibrada, ya que los individuos de clases altas, eliminados ocasionalmente, son remplazados por los existentes en las clases inferiores (Rollet 1980, Lamprecht 1990). Sin embargo, el hecho de que las distribuciones no presenten árboles de clases pequeñas $(1 \leq \mathrm{D}<$ $10 \mathrm{~cm}$ ) sugiere que la regeneración del roble puede estar condicionada por factores microambientales que de no ser favorables, pondrían en peligro la persistencia de esta especie; al respecto, algunos autores afirman que el roble, como muchas otras especies que alcanzan el dosel en bosques tropicales húmedos y muy húmedos, requieren la existencia de aperturas en el dosel para su regeneración (Clark \& Clark 1987). Las diferencias en tamaños máximos alcanzados por los árboles de roble en las parcelas, podrían ser el resultado de la fertilidad de los suelos. Por ejemplo, el alto valor de diámetro registrado en San Andrés de Cuerquia, coincide con el mayor nivel de fertilidad de esta parcela, donde la materia orgánica y los niveles de fósforo son superiores a los de las parcelas restantes. Estos resultados, permiten concluir que las parcelas presentan diferencias sutiles en sus estructuras diamétricas como resultados de las características particulares del sitio, especialmente la fertilidad del suelo; resultados análogos se han documentado en bosques tropicales de tierras bajas, donde los usos previos del suelo y las características de los mismos condicionan la estructura diamétrica de los bosques así como la composición florística (Saldarriaga 1991, Aide et al. 1995, Finegan 1996, Ferreira \& Prance 1999, Kennard 2001). En general, aunque los ecosistemas estudiados presentan características biofísicas similares y corresponden a relictos de bosques cuya especie dominante es el roble, las especies asociadas y la abundancia e importancia de las mismas, varía de acuerdo con características de los sitios, que se ven reflejadas en parámetros estructurales como la composición florística y la estructura diamétrica.

En resumen, los resultados permiten concluir que los tres robledales estudiados difieren en su composición florística y estructura a pesar de presentar características biofísicas similares. Las diferencias encontradas se deben posiblemente, a los diferentes factores que interactúan en cada sitio como la historia de uso, la intensidad y duración de las perturbaciones antrópicas pasadas, la topografía, la fauna y el clima local, que se ven reflejados en los parámetros estructurales de la vegetación.

\section{AGRADECIMIENTOS}

A CORANTIOQUIA por la financiación del estudio. A Cipreses de Colombia S.A., y a Gustavo Mazo, por proporcionar los predios para el establecimiento de las parcelas permanentes y el apoyo logístico. A Fernando Ocampo $(\dagger)$ y Juan Lázaro Toro por su colaboración en el establecimiento de las parcelas y la identificación del material botánico, respectivamente. Finalmente, a Álvaro Lema Tapias por su asesoría estadística.

\section{RESUMEN}

La alta diversidad florística que albergan los ecosistemas andinos está siendo amenazada por fuertes presiones de origen antrópico. Uno de estos ecosistemas son los robledales, bosques dominados por la especie Quercus humboldtii, que se encuentra seriamente amenazada en Colombia porque su madera es valiosa comercial y dendroenergéticamente. En este estudio, se caracterizó la composición florística y la estructura de tres robledales de los Andes colombianos, con el fin de determinar su similaridad. En cada sitio, se estableció una parcela permanente $\left(5000 \mathrm{~m}^{2}\right)$ donde se muestrearon todos los árboles con $D$ $\geq 10 \mathrm{~cm}$. En los tres sitios, la especie dominante fue $Q$. humboldtii, pero la similitud florística entre ellos no fue alta. La estructura por clases de tamaño para los tres sitios fue en forma de $\mathrm{J}$ invertida, aunque varió la abundancia 
de individuos por clase diamétrica. Los resultados indican que los robledales estudiados difieren en su composición florística y estructura a pesar de presentar características biofísicas similares. Las diferencias encontradas se deben posiblemente, a los diferentes factores que interactúan en cada sitio como la historia de uso, intensidad y duración de las perturbaciones antrópicas pasadas, topografía, fauna y clima local, que se ven reflejados en los parámetros estructurales de la vegetación.

Palabras clave: bosque andino, composición florística, estructura, Quercus humboldtii, riqueza, robledal.

\section{REFERENCIAS}

Aide, T.M., J.K. Zimmerman, L. Herrera \& M. Rosario. 1995. Forest recovery in abandoned tropical pastures in Puerto Rico. For. Ecol. Manag. 77: 77-86.

Becerra, J.E. 1989. Estructura y crecimiento de un bosque secundario de roble (Quercus humboldtii Bonpl.). Rev. Col. For. 3: 1-64.

Brown, A.D. \& M. Kappelle. 2001. Introducción a los bosques nublados del geotrópico: una síntesis regional, p. 25-40. In M. Kappelle \& A.D. Brown (eds.). Bosques nublados del geotrópico. Instituto Nacional de Biodiversidad (INBIO). Santo Domingo de Heredia, Costa Rica.

Castaño, G. \& J.C. Patiño. 2000. Cambios en la composición de avifauna en Santa Elena durante el siglo XX. Cron. For. Amb. 15: 139-163.

Cavelier, J., D. Lizcano \& M.T. Pulido. 2001. Structure and Composition of Colombian Montane Oak Forests, p. 25-40. In M. Kappelle \& A.D. Brown (eds.). Bosques nublados del geotrópico. Instituto Nacional de Biodiversidad (INBIO). Santo Domingo de Heredia, Costa Rica.

Churchill, S.P., H. Balslev, E. Forero \& J.L. Luteyn. 1995. Biodiversity and conservation of neotropical montane forest. The New York Botanical Garden, New York, EEUU.

Clark, D.A. \& D.B. Clark. 1987. Spacing dynamics of a tropical rain forest tree: evaluation of the JanzenConell Model. Am. Nat. 24: 769-788.

Coomes, D.A. \& R.B. Allen. 2007. Mortality and tree-size distributions in natural mixed-age forest. J. Ecol. 95: $27-40$.

Cuatrecasas, J. 1958. Aspectos de la vegetación natural en Colombia. Rev. Acad. Colomb. Cienc. 10: 221-268.
Denslow, J.S. 1980. Gap partitioning among tropical rainforest trees. Biotropica 12: 47-55.

Etter, A., C. Mcalpine, K. Wilson, S. Phinn \& H. Possingham. 2006. Regional patterns of agricultural land use and deforestation in Colombia. Agr. Ecosyst. Environ. 114: 369-386.

Ferreira, L.V. \& G.T. Prance. 1999. Ecosystem recovery in terra firme forest after cutting and burning: a comparison on species richness, floristic composition and forest structure in the Jaú National Park, Amazonia. Bot. J. Linn. Soc. 130: 97-110.

Finegan, B. 1996. Pattern and process in neotropical secondary rain forest: the first 100 years of succession. Trens. Ecol. Evol. 11: 119-124.

Fontaine, R.G., A. Gómez-Pompa \& B. Ludlow. 1980. Sucesiones secundarias, p. 245-263. In UNESCO, UNEP \& FAO (eds.). Ecosistemas de los bosques tropicales: Informe sobre el estado de conocimientos. Serie de Investigaciones sobre los Recursos Naturales XVI. Madrid, España.

Galindo, R., J. Betancur. \& J.J. Cadena. 2003. Estructura y composición florística de cuatro bosques andinos del santuario de flora y fauna Guanentá-Alto río Fonce, cordillera oriental colombiana. Caldasia 25: 313-335.

Gentry, A. 1995. Patterns of diversity and floristic composition in Neotropical Montane Forests, p. 103-126. In S. Churchill, H. Balslev, E. Forero \& J. Luteyn (eds.). Biodiversity and Conservation of Neotropical Montane Forests. The New York Botanical Garden, Bronx. New York, EEUU.

Guariguata, M.R. \& G.R. Ostertag. 2001. Neotropical secondary forest successions: changes in structural and functional characteristics. For. Ecol. Manag. 148: 185-206.

Hartshorn, G.S. 1980. Neotropical forest dynamics. Biotropica 12: 23-30.

Henderson, A.S.P., S.P. Churchil \& J.L. Luteyn. 1991. Neotropical plan diversity. Nature 351: 21-22.

IDEADE. 1996. Evaluación de estatus ecosistémico y manejo de los bosques de Fagaceas (Quercus humboldtii Bonpl. y Trigonobalanus excelsus) en el norte de la cordillera oriental (Cundinamarca, Santander y Boyacá). Informe Final. Universidad Pontificia Bolivariana, Santa Fe de Bogotá, Colombia. 
Kappelle, M. 2001. Costa Rica, p. 301-370. In M. Kappelle \& A.D. Brown (eds.). Bosques nublados del geotrópico. Instituto Nacional de Biodiversidad (INBIO). Santo Domingo de Heredia, Costa Rica.

Kennard, K.K. 2001. Secondary forest succession in a tropical dry forest: patterns of development across a 50-year chronosequence in lowland Bolivia. J. Trop. Ecol. 18: 53-66.

Lamprecht, H. 1990. Silvicultura en los trópicos: los ecosistemas forestales en los bosques tropicales y sus especies arbóreas-posibilidades y métodos para un aprovechamiento sostenido. (GTZ) GMBH, Eschborn, Alemania.

León, J.D. \& E. Giraldo. 2000. Crecimiento diamétrico en robledales del norte y centro de Antioquia, Colombia. Cron. For. Amb. 15: 121-138.

León, J.D. 2008. Ecología de bosques andinos: experiencias de investigación. La Carreta, Medellín, Colombia.

Maleheiros, D.E.O. \& E. Rotta. 1982. Levantamiento horizontal de uma mata de araucaria doprimeiro planalto paranaense. Bol. Pesqui. Flor. 4: 1-39.

Marín-Corba, C. \& J. Betancur. 1997. Estudio florístico en un robledal del santuario de flora y fauna de Iguaqué (Boyacá, Colombia). Rev. Acad. Colomb. Cienc. 21: 249-259.

Martínez-Ramos, M. 1985. Claros, ciclos vitales de los árboles tropicales y regeneración natural de las selvas altas perennifolias, p. 191-221. In A. GómezPompa \& S. Amo (eds). Investigaciones sobre la regeneración de selvas altas en Veracruz, México. Continental, México.

Matteucci, S.D. \& A. Colma. 1982. Metodología para el estudio de la vegetación. Secretaría General de la Organización de los Estados Americanos. Washington, EEUU.

Pulido, M.T., J. Cavelier \& S.P. Cortés. 2006. Structure and composition of colombian montane oak forest, p: 141-151. In M. Kappelle (ed.). Ecology and Conservation of Neotropical Montane Oak Forests. Springer-Verlag, Berlin, Heidelberg, Alemania.

Rollet, B. 1980. Organización, p. 126-154. In UNESCO, UNEP \& FAO (eds.). Ecosistemas de los bosques tropicales: Informe sobre el estado de conocimientos. Serie de Investigaciones sobre los Recursos Naturales XVI, Madrid, España.

Saldarriaga, J.G. 1991. Estudios en la Amazonía colombiana V: Recuperación de la Selva de "Tierra Firme" en el alto río Negro Amazonía colombiana-venezolana. TROPENBOS Colombia. Bogotá, Colombia.

Vélez, G. \& E. Fresneda. 1992. Diversidad florística en las comunidades robledal y rastrojo alto en la cuenca de la quebrada Piedras Blancas, Antioquia. Rev. Fac. Nal. Agr. 45: 3-25.

Vélez, N. \& S. Botero. 2000. La búsqueda del Valle Arví. Corantioquia. Medellín, Colombia. 
ANEXO 1

Familias, géneros y especies encontradas en robledales de la Cordillera Central de Antioquia (Colombia)

ANNEX 1

Families, genus and species found in colombian Andean oak forests

\begin{tabular}{|c|c|c|}
\hline \multicolumn{3}{|c|}{ San Andrés de Cuerquia } \\
\hline \multicolumn{3}{|c|}{$\mathrm{D}>10 \mathrm{~cm}(0.5 \mathrm{ha})$} \\
\hline Familia & Género & Especie \\
\hline Fagaceae & Quercus & Quercus humboldtii Bonpl. \\
\hline Clusiaceae & Clusia & Clusia sp. \\
\hline Melastomataceae & Tibouchina & Tibouchina lepidota (Bonpl.) Baill. \\
\hline Myrsinaceae & Myrsine & $\begin{array}{l}\text { Myrsine coriacea } \\
\text { (Sw.) R. Br. ex Roem. \& Schult. }\end{array}$ \\
\hline Araliaceae & Schefflera & Schefflera $\mathrm{sp}$ \\
\hline Ericaceae & Cavendishia & Cavendishia pubescens (Kunth) Hemsl. \\
\hline Clethraceae & Clethra & Clethra fagifolia Kunth. \\
\hline Clusiaceae & Clusia & Clusia sp1. \\
\hline Winteraceae & Drimys & Drimys granadensis L. f. \\
\hline Cunnoniaceae & Weinmannia & Weinmannia pubescens Kunth \\
\hline \multicolumn{3}{|c|}{$5 \leq \mathrm{D}<10 \mathrm{~cm}(0.05 \mathrm{ha})$} \\
\hline Melastomataceae & Miconia & Miconia sp1. \\
\hline Fagaceae & Quercus & Quercus humboldtii Bonpl. \\
\hline Araliaceae & Schefflera & Schefflera sp. \\
\hline Clusiaceae & Clusia & Clusia sp. \\
\hline Melastomataceae & Miconia & Miconia sp. \\
\hline Cunnoniaceae & Weinmannia & Weinmannia pubescens Kunth \\
\hline \multicolumn{3}{|c|}{$2 \leq \mathrm{D}<5 \mathrm{~cm}(0.0144 \mathrm{ha})$} \\
\hline Ericaceae & Cavendishia & Cavendishia pubescens (Kunth) Hemsl. \\
\hline Rubiaceae & Graffenrieda & Graffenrieda sp. \\
\hline Melastomataceae & Miconia & Miconia sp1. \\
\hline Monimiaceae & Monnina & Monnina sp. \\
\hline Clusiaceae & Clusia & Clusia sp. \\
\hline Winteraceae & Drimys & Drimys granadensis L. f. \\
\hline Euphorbiaceae & Hyeronima & Hyeronima sp. \\
\hline Melastomataceae & Meriniana & Meriniana sp. \\
\hline Myrsinaceae & Myrsine & $\begin{array}{l}\text { Myrsine coriacea } \\
\text { (Sw.) R. Br. ex Roe }\end{array}$ \\
\hline Fagaceae & Quercus & Quercus humboldtii Bonpl. \\
\hline \multicolumn{3}{|c|}{ Belmira } \\
\hline \multicolumn{3}{|c|}{$\mathrm{D}>10 \mathrm{~cm}(0.5 \mathrm{ha})$} \\
\hline Familia & Género & Especie \\
\hline Fagaceae & Quercus & Quercus humboldtii Bonpl. \\
\hline Melastomataceae & Meriniana & Meriniana sp. \\
\hline Clethraceae & Clethra & Clethra fagifolia Kunth. \\
\hline Cunoniaceae & Weinmannia & Weinmannia pubescens Kunth \\
\hline Melastomataceae & Tibuchina & Tibouchina lepidota (Bonpl.) Baill. \\
\hline Aquifoliaceae & Ilex & Ilex laurina Kunth \\
\hline Araliaceae & Schefflera & Schefflera sp1. \\
\hline & Ternstroemia & Ternstroemia $\mathrm{sp}$. \\
\hline
\end{tabular}


ANEXO 1 (Continuación)

Familias, géneros y especies encontradas en robledales de la Cordillera Central de Antioquia (Colombia)

ANNEX 1 (Continue)

Families, genus and species found in colombian Andean oak forests

\begin{tabular}{|c|c|c|}
\hline Clusiaceae & Clusia & Clusia sp1. \\
\hline Theaceae & Gordonia & Gordonia fruticosa (Schrad.) H. Keng \\
\hline Rubiaceae & Ladenbergia & Ladenbergia sp. \\
\hline Myrsinaceae & Myrsine & Myrsine coriacea (Sw.) R. Br. ex Roe \\
\hline Melastomataceae & Miconia & Miconia $\mathrm{sp} 51$ \\
\hline Brunelliaceae & Brunellia & Brunellia sp. \\
\hline Melastomataceae & Miconia & Miconia sp2. \\
\hline Rubiaceae & Palicourea & Palicourea sp1. \\
\hline Lauraceae & Persea & Persea chrysophylla L.E. Kopp \\
\hline \multicolumn{3}{|c|}{$5 \leq \mathrm{D}<10 \mathrm{~cm}(0.05 \mathrm{ha})$} \\
\hline Rubiaceae & Psychotria & Psychotria sp. \\
\hline Rubiaceae & Psychotria & Psychotria sp. \\
\hline Rubiaceae & Palicourea & Palicourea sp. \\
\hline Melastomataceae & Miconia & Miconia sp1. \\
\hline Melastomataceae & Miconia & Miconia sp2. \\
\hline Melastomataceae & Miconia & Miconia sp3. \\
\hline Melastomataceae & Miconia & Miconia sp4. \\
\hline Melastomataceae & Miconia & Miconia sp5. \\
\hline Rhamnaceae & Rhamnus & Rhamnus goudotiana Triana \& Planch. \\
\hline Cunoniaceae & Weinmannia & Weinmannia pubescens Kunth \\
\hline \multicolumn{3}{|c|}{ Guarne } \\
\hline \multicolumn{3}{|c|}{$\mathrm{D}>10 \mathrm{~cm}(0.5 \mathrm{ha})$} \\
\hline Familia & Género & Especie \\
\hline Fagaceae & Quercus & Quercus humboldtii Bonpl. \\
\hline Melastomataceae & Myrcia & Myrcia popayanensis Hieron. \\
\hline Juglandaceae & Alfaroa & Alfaroa colombiana Lozano, Hern. Cam. \& Espinal \\
\hline Lauraceae & Nectandra & Nectandra sp. \\
\hline Saxifragaceae & Escallonia & Escallonia paniculata (Ruiz \& Pav.) Roem. \& Schult. \\
\hline Clusiaceae & Vismia & Vismia sp. \\
\hline Aquifoliaceae & Ilex & Ilex laurina Kunth \\
\hline Proteaceae & Panopsis & Panopsis yolombo (Pos.-Arang.) Killip \\
\hline Clethraceae & Clethra & Clethra fagifolia Kunth. \\
\hline Cunnoniaceae & Wainmannia & Weinmannia pubescens Kunth \\
\hline Euphorbiaceae & Alchornea & Alchornea sp. \\
\hline Lecythidaceae & Eschweilera & Eschweilera antioquensis Dugand \& Daniel \\
\hline Euphorbiaceae & Hyeronima & Hyeronima antioquensis \\
\hline Fabaceae & Ormosia & Ormosia sp. \\
\hline Proteaceae & Roupala & Roupala grandifolia Klotzsch \\
\hline Myrtaceae & Eugenia & Eugenia sp. \\
\hline Theaceae & Frezieria & Frezieria sp. \\
\hline Rubiaceae & Guettarda & Guettarda sp. \\
\hline Sapindaceae & Matayba & Matayba sp. \\
\hline Symplocaceae & Symplocos & Symplocos serrulata Bonpl. \\
\hline Lauraceae & Aiouea & Aiouea sp. \\
\hline
\end{tabular}


ANEXO 1 (Continuación)

Familias, géneros y especies encontradas en robledales de la Cordillera Central de Antioquia (Colombia)

ANNEX 1 (Continue)

Families, genus and species found in colombian Andean oak forests

\begin{tabular}{|c|c|c|}
\hline Araliaceae & Dendropanax & Dendropanax sp. \\
\hline Myrsinaceae & Geissanthus & Geissanthus sp. \\
\hline Indeterminada & Indeterminada & Indeterminada 1. \\
\hline Elaeocarpaceae & Sloanea & Sloanea sp. \\
\hline Ericaceae & Befaria & Befaria glauca Bonpl. \\
\hline Chloranthaceae & Hedyosmum & Hedyosmum bomplandianum Kunth \\
\hline Aquifoliaceae & Ilex & Ilex sp. \\
\hline Indeterminada & Indeterminada & Indeterminada 2. \\
\hline Indeterminada & Indeterminada & Indeterminada 3. \\
\hline Mimosaceae & Inga & Inga archeri Britton \& Killip \\
\hline Mimosaceae & Inga & Inga sp2. \\
\hline Myrtaceae & Myrcia & Myrcia sp. \\
\hline Myrsinaceae & Myrsine & Myrsine coriacea (Sw.) R. Br. ex Roem. \& Schult. \\
\hline Rubiaceae & Palicourea & Palicourea sp. \\
\hline Araliaceae & Schefflera & Schefflera uribei Cuatrec. \\
\hline Clusiaceae & Tovomitopsis & Tovomitopsis sp. \\
\hline \multicolumn{3}{|c|}{$5 \leq \mathrm{D}<10 \mathrm{~cm}(0.05 \mathrm{ha})$} \\
\hline Myrtaceae & Myrcia & Myrcia sp. \\
\hline Symplocaceae & Symplocos & Symplocos serrulata Bonpl. \\
\hline Rubiaceae & Palicourea & Palicourea sp. \\
\hline Juglandaceae & Alfaroa & Alfaroa colombiana Lozano, Hern. Cam. \& Espinal. \\
\hline Myrtaceae & Eugenia & Eugenia sp. \\
\hline Caprifoliaceae & Viburnum & Viburnum cordifolium Wall. ex DC. \\
\hline Euphorbiaceae & Alchornea & Alchornea sp1. \\
\hline Boraginaceae & Cordia & Cordia archeri \\
\hline Myrtaceae & Myrcia & Myrcia popayanensis Hieron \\
\hline Fabaceae & Ormosia & Ormosia sp. \\
\hline Piperaceae & Piper & Piper archeri Trel. \& Yunck. \\
\hline Euphorbiaceae & Alchornea & Alchornea sp. \\
\hline Araliaceae & Dendropanax & Dendropanax sp. \\
\hline Myrsinaceae & Geissanthus & Geissanthus sp. \\
\hline Myrsinaceae & Geissanthus & Geissanthus kalbreyeri $\mathrm{Mez}$ \\
\hline Aquifoliaceae & Ilex & Ilex sp. \\
\hline Indeterminada & Indeterminada & Indeterminada \\
\hline Lauraceae & Nectandra & Nectandra sp. \\
\hline Proteaceae & Panopsis & Panopsis yolombo (Pos.-Arang.) Killip \\
\hline Proteaceae & Roupala & Roupala grandifolia Klotzsch \\
\hline Rubiaceae & Sarauia & Sarauia sp. \\
\hline Symplocaceae & Symplocos & Symplocos rigidissima Brand \\
\hline Staphileaceae & Turpinia & Turpinia heterophylla (Ruiz \& Pav.) Tul. \\
\hline Caprifoliaceae & Viburnum & Viburnum anabaptista Graebn. \\
\hline Clusiaceae & Vismia & Vismia sp. \\
\hline Cunnoniacea & Weinmannia & Weinmannia pubescens Kunth \\
\hline
\end{tabular}


ANEXO 1 (Continuación)

Familias, géneros y especies encontradas en robledales de la Cordillera Central de Antioquia (Colombia)

ANNEX 1 (Continue)

Families, genus and species found in colombian Andean oak forests

$2 \leq \mathrm{D}<5 \mathrm{~cm}(0.0144 \mathrm{ha})$

$\begin{array}{lll}\text { Juglandaceae } & \text { Alfaroa } & \text { Alfaroa colombiana Alfaroa colombiana Lozano, Hern. } \\ \text { Mimosaceae } & \text { Inga } & \text { Cam. \& Espinal } \\ \text { Ericaceae } & \text { Cavendischia } & \text { Cavendishia } \text { sp. } \\ \text { Euphorbiaceae } & \text { Hyeronima } & \text { Hyeronima antioquensis } \\ \text { Indeterminada } & \text { Indeterminada } & \text { Indeterminada } 1 . \\ \text { Indeterminada } & \text { Indeterminada } & \text { Indeterminada } 2 . \\ \text { Melastomataceae } & \text { Cavendischia } & \text { Cavendishia pubescens } \text { (Kunth) Hems } 1 \\ \text { Ericaceae } & \text { Clusia } & \text { Clusia } \text { sp1. } \\ \text { Boraginaceae } & \text { Cordia } & \text { Cordia } \text { archeri } \\ \text { Araliaceae } & \text { Dendropanax } & \text { Dendropanax } \text { sp. } \\ \text { Myrsinaceae } & \text { Geissanthus } & \text { Geissanthus } \text { sp. } \\ \text { Aquifoliaceae } & \text { Ilex } & \text { Ilex laurina } \text { Kunth } \\ \text { Indeterminada } & \text { Indeterminada } & \text { Indeterminada } 3 . \\ \text { Sapindaceae } & \text { Matayba } & \text { Matayba } \text { sp. } \\ \text { Melastomataceae } & \text { Miconia } & \text { Miconia } \text { sp2. }\end{array}$


ANEXO 2

Índice de valor de importancia (IVI) para robledales de la Cordillera Central de Antioquia (Colombia)

ANNEX 2

Importance Value Index (IVI) for colombian Andean oak forests

\begin{tabular}{|c|c|c|c|c|c|c|c|c|c|c|c|c|}
\hline \multirow{2}{*}{ Especie } & \multicolumn{4}{|c|}{ San Andrés de Cuerquia } & \multicolumn{4}{|c|}{ Guarne } & \multicolumn{4}{|c|}{ Belmira } \\
\hline & DeR & FR & DoR & IVI & DeR & FR & DoR & IVI & DeR & FR & DoR & IVI \\
\hline Aiouea sp. & & & & & 0.73 & 2.06 & 1.89 & 4.68 & & & & \\
\hline Alchornea sp. & & & & & 1.46 & 4.12 & 0.66 & 6.24 & & & & \\
\hline $\begin{array}{l}\text { Alfaroa colombiana Lozano, Hern. } \\
\text { Cam. \& Espinal. }\end{array}$ & & & & & 4.01 & 4.12 & 1.33 & 9.46 & & & & \\
\hline Befaria glauca Bonpl. & & & & & 0.36 & 1.03 & 0.10 & 1.49 & & & & \\
\hline Brunellia sp. & & & & & & & & & 0.29 & 1.96 & 0.19 & 2.44 \\
\hline $\begin{array}{l}\text { Cavendishia pubescens (Kunth) } \\
\text { Hemsl }\end{array}$ & 0.61 & 4.55 & 0.14 & 5.30 & & & & & & & & \\
\hline Clethra fagifolia Kunth & 0.61 & 4.55 & 0.10 & 5.26 & 1.82 & 4.12 & 0.86 & 6.80 & 3.24 & 11.76 & 2.47 & 17.47 \\
\hline Clusia sp. & 1.81 & 9.09 & 0.92 & 11.82 & & & & & 0.88 & 3.92 & 0.24 & 5.04 \\
\hline Clusia sp1. & 0.61 & 4.55 & 0.12 & 5.28 & & & & & & & & \\
\hline Dendropanax sp. & & & & & 0.73 & 2.06 & 0.30 & 3.09 & & & & \\
\hline Drimys granadensis L. f. & 0.61 & 4.55 & 0.42 & 5.58 & & & & & & & & \\
\hline $\begin{array}{l}\text { Escallonia paniculata (Ruiz \& Pav.) } \\
\text { Roem. \& Schult. }\end{array}$ & & & & & 3.65 & 5.15 & 1.97 & 10.77 & & & & \\
\hline $\begin{array}{l}\text { Eschweilera antioquensis Dugand } \\
\text { \& Daniel }\end{array}$ & & & & & 1.46 & 2.06 & 0.39 & 3.91 & & & & \\
\hline Eugenia sp. & & & & & 1.09 & 1.03 & 0.41 & 2.53 & & & & \\
\hline Freziera $\mathrm{sp}$. & & & & & 1.09 & 1.03 & 0.31 & 2.43 & & & & \\
\hline Geissanthus sp. & & & & & 0.73 & 2.06 & 0.18 & 2.97 & & & & \\
\hline $\begin{array}{l}\text { Gordonia fruticosa (Schrad.) } \\
\text { H. Keng }\end{array}$ & & & & & & & & & 0.88 & 3.92 & 0.42 & 5.22 \\
\hline Guettarda sp. & & & & & 1.09 & 2.06 & 1.16 & 4.31 & & & & \\
\hline Hedyosmum bomplandianum Kunth & & & & & 0.36 & 1.03 & 0.09 & 1.48 & & & & \\
\hline Hyeronima antioquensis & & & & & 1.46 & 2.06 & 1.18 & 4.70 & & & & \\
\hline Ilex laurina Kunth & & & & & 3.28 & 6.19 & 1.81 & 11.28 & & & & \\
\hline Ilex sp. & & & & & 0.36 & 1.03 & 0.08 & 1.47 & 1.47 & 1.96 & 0.69 & 4.12 \\
\hline Indeterminada 1 & & & & & 0.73 & 1.03 & 0.34 & 2.10 & & & & \\
\hline Indeterminada 2 & & & & & 0.36 & 1.03 & 0.28 & 1.67 & & & & \\
\hline Indeterminada 3 & & & & & 0.36 & 1.03 & 0.28 & 1.67 & & & & \\
\hline Inga archeri Britton \& Killip & & & & & 0.36 & 1.03 & 1.26 & 2.65 & & & & \\
\hline Inga sp2. & & & & & 0.36 & 1.03 & 0.08 & 1.47 & & & & \\
\hline Ladenbergia sp. & & & & & & & & & 0.88 & 5.88 & 0.42 & 7.18 \\
\hline Matayba sp. & & & & & 1.09 & 3.09 & 0.27 & 4.45 & & & & \\
\hline Meriniana sp. & & & & & & & & & 5.29 & 7.84 & 2.51 & 15.64 \\
\hline Miconia sp2. & & & & & & & & & 0.29 & 1.96 & 0.15 & 2.40 \\
\hline Miconia sp5. & & & & & & & & & 0.59 & 3.92 & 0.31 & 4.82 \\
\hline Myrcia popayanensis Hieron & & & & & 5.47 & 8.25 & 2.72 & 16.44 & & & & \\
\hline Myrcia sp. & & & & & 0.36 & 1.03 & 0.19 & 1.58 & & & & \\
\hline $\begin{array}{l}\text { Myrsine coriacea } \\
\text { (Sw.) R. Br. ex Roe }\end{array}$ & 1.23 & 9.09 & 1.26 & 11.58 & 0.36 & 1.03 & 0.32 & 1.71 & 0.88 & 3.92 & 0.42 & 5.22 \\
\hline Nectandra sp1. & & & & & 4.01 & 4.12 & 1.30 & 9.43 & & & & \\
\hline
\end{tabular}


ANEXO 2 (Continuación)

Índice de valor de importancia (IVI) para robledales de la Cordillera Central de Antioquia (Colombia)

ANNEX 2 (Continued)

Importance Value Index (IVI) for colombian Andean oak forests

\begin{tabular}{|c|c|c|c|c|c|c|c|c|c|c|c|c|}
\hline \multirow{2}{*}{ Especie } & \multicolumn{4}{|c|}{ San Andrés de Cuerquia } & \multicolumn{4}{|c|}{ Guarne } & \multicolumn{4}{|c|}{ Belmira } \\
\hline & DeR & FR & DoR & IVI & DeR & FR & DoR & IVI & DeR & FR & DoR & IVI \\
\hline Ormosia sp. & & & & & 1.46 & 2.06 & 0.62 & 4.14 & & & & \\
\hline Palicourea sp. & & & & & 0.36 & 1.03 & 0.12 & 1.51 & 0.29 & 1.96 & 0.18 & 2.43 \\
\hline $\begin{array}{l}\text { Panopsis yolombo (Pos.-Arang.) } \\
\text { Killip }\end{array}$ & & & & & 2.19 & 5.15 & 0.64 & 7.98 & & & & \\
\hline Persea chrysophylla L.E. Kopp & & & & & & & & & 0.29 & 1.96 & 0.12 & 2.37 \\
\hline Quercus humboldtii Bonpl. & 90.80 & 45.46 & 95.30 & 231.56 & 49.27 & 10.31 & 74.19 & 133.77 & 77.65 & 19.61 & 86.17 & 183.43 \\
\hline Roupala grandifolia Klotzsch & & & & & 1.46 & 3.09 & 0.46 & 5.01 & & & & \\
\hline Schefflera $\mathrm{sp}$. & 1.23 & 9.09 & 0.52 & 10.84 & & & & & & & & \\
\hline Shefflera sp1. & & & & & & & & & 1.47 & 7.84 & 1.37 & 10.68 \\
\hline Schefflera uribei Cuatrec. & & & & & 0.36 & 1.03 & 0.11 & 1.50 & & & & \\
\hline Sloanea sp. & & & & & 0.73 & 1.03 & 0.29 & 2.05 & & & & \\
\hline Symplocos serrulata Bonpl. & & & & & 1.09 & 2.06 & 1.69 & 4.84 & & & & \\
\hline Ternstroemia sp. & & & & & & & & & 1.47 & 7.84 & 0.84 & 10.15 \\
\hline Tibouchina lepidota (Bonpl.) Baill. & 1.84 & 16.58 & 1.08 & 19.50 & & & & & 1.76 & 3.92 & 1.69 & 7.37 \\
\hline Tovomitopsis sp. & & & & & 0.36 & 1.03 & 0.12 & 1.51 & & & & \\
\hline Vismia $\mathrm{sp}$. & & & & & 3.65 & 5.15 & 1.32 & 10.12 & & & & \\
\hline Weinmannia pubescens Kunth & 0.61 & 4.55 & 0.14 & 5.30 & 1.82 & 4.12 & 0.68 & 6.62 & 2.35 & 9.80 & 1.81 & 13.96 \\
\hline
\end{tabular}

